\title{
EWVDV-Mediated Platelet-Targeting Nanoparticles for the Multimodal Imaging of Thrombi at Different Blood Flow Velocities
}

This article was published in the following Dove Press journal: International Journal of Nanomedicine

\author{
Jie $X u^{\prime}$ \\ Jun Zhou' \\ Yixin Zhong' \\ Yu Zhang $\mathbb{D}^{\prime}$ \\ Man Ye' \\ Jingxin Hou' \\ Zhigang Wang ${ }^{2}$ \\ Haitao Ran ${ }^{2}$ \\ Jia Liu' \\ Dajing Guo'
}

'Department of Radiology, The Second Affiliated Hospital of Chongqing Medical University, Chongqing, People's Republic of China; ${ }^{2}$ Institute of Ultrasound Imaging, Department of Ultrasound, The Second Affiliated Hospital of Chongqing Medical University, Chongqing, People's Republic of China
Correspondence: Dajing Guo; Jia Liu Department of Radiology, The Second Affiliated Hospital of Chongqing Medical University, No. 74 Linjiang Road, Yuzhong District, Chongqing 4000 I0, People's Republic of China

Tel +86236369378I

Email guodaj@163.com;

442775883@qq.com
Background: There have been many recent reports of molecular probes for thrombi but with unsatisfactory in vivo targeting effects, which could be related to the blood flow velocity in vivo. Therefore, it is worth explaining the relationship between the targeting effect and the blood flow velocity.

Methods and Materials: In this study, we constructed a platelet-targeting nanoparticle (NP) based on EWVDV for targeting P-selectin combined with the phase transition material perfluorohexane and India ink to achieve the multimodal imaging of thrombi. We studied the targeting effect of the NPs for rabbit blood thrombi under different flow velocities simulating blood flow velocities in vivo.

Results: The results show the successful fabrication of NPs with the ability to undergo a phase transition via low-intensity focused ultrasound irradiation to achieve ultrasound imaging and with a high binding affinity for activated platelets. In vitro, low flow velocities $(20 \mathrm{~cm} / \mathrm{s})$ hardly affected the targeting effect of the NPs, while moderate flow velocities $(40 \mathrm{~cm} / \mathrm{s})$ reduced the number of NPs that target thrombi by $52.6 \%$ comparing to static fluid $(0 \mathrm{~cm} / \mathrm{s})$. High flow velocities $(60 \mathrm{~cm} / \mathrm{s})$ greatly reduced the targeting effect of the NPs by $83.5 \%$.

Conclusion: These results can serve as a reference for the design of NPs targeting thrombi at different sites and in different blood vessel types according to the blood flow velocity, thereby establishing a foundation for in vivo experiments.

Keywords: phase transition, thrombus, multimodal imaging, blood flow velocity

\section{Introduction}

Thrombotic disease is a serious threat to human health. Ischemic heart disease and stroke were the two main causes of premature mortality in the world, affecting 15.2 million people in 2015 and accounting for $85.1 \%$ of all deaths due to cardiovascular disease that year. ${ }^{1}$ Early and accurate diagnosis and timely treatment can rescue ischemic tissue and reduce complications and mortality.

Molecular probes have distinct advantages for the early diagnosis and treatment of thrombi due to their unique biological approach to the targeted delivery of contrast agents or thrombolytic drugs. ${ }^{2}$ At present, various types of thrombustargeted molecular probes, such as those for magnetic resonance imaging (MRI), ${ }^{3}$ nuclear imaging, ${ }^{4}$ ultrasound (US), ${ }^{5}$ micro-computed tomography (CT), ${ }^{6}$ optical imaging, ${ }^{7}$ photoacoustic (PA) imaging ${ }^{8}$ and multimodal imaging, ${ }^{9}$ have been reported and achieved encouraging results in the early diagnosis and treatment of 
thrombi. However, the targeting effect of these molecular probes for thrombi in vivo is not completely satisfactory, particularly for arterial thrombi. ${ }^{10}$ Many factors influence the adhesion of targeted NPs to a thrombus, mainly including ligand affinity, carrier features, target features, target microenvironment and NP behavior in circulation. ${ }^{11}$ Among such factors, the blood flow velocity may also be important. Because in addition to the thrombus components, the rapid blood flow velocity in arteries is a distinct feature compared with veins.

In the field of basic research, micro- and nanoscale devices have been used for researching the influence of shear force on the targeting effect of molecular probes at the cellular level and have revealed the relationship between shear force and targeted effects at the microscopic scale. ${ }^{10,12-14}$ These studies are very meaningful and facilitate the understanding of targeted delivery mechanisms. However, most of these studies only selected one cellular component of thrombi, which is very different from actual thrombi. Furthermore, in clinical work, it is difficult to determine blood shear forces in blood vessels due to technical limitations, but the blood flow velocity could be directly measured by common clinical imaging modalities, such as US and MRI. ${ }^{15,16}$ Therefore, revealing the relationship between the targeting effect of NPs and the blood flow velocity may be more helpful for clinical application.

Compared with single-modal imaging probes, multimodal molecular probes can provide abundant and accurate information, which is very useful for the visualization and specific identification of thrombi. ${ }^{9}$ Furthermore, multimodal NPs can provide multiple options for doctors according to the clinical situation to satisfy the diverse and personalized needs of patients. Perfluorohexane (PFH) has good stability and biosafety in combination with low-intensity focused ultrasound (LIFU)-induced phase transition technology for US imaging. ${ }^{17}$ India ink mainly consists of carbon black particles, which exhibit strong absorption in the visible and near-infrared regions and have the ability to provide excellent PA contrast. ${ }^{18}$ In our previous work, we constructed novel multimodal NPs to detect thrombi and found obvious advantages of multimodal imaging in detecting thrombi; additionally, the NPs were smaller in size due to the introduction of $\mathrm{PFH}$, allowing them to penetrate thrombi well. ${ }^{19}$ These NPs can easily avoid capture by the reticuloendothelial system, show prolonged blood circulation, and can more easily attach to thrombi under high shear stress. ${ }^{17,20}$
In the present study, we chose poly (lactic-co-glycolic acid) (PLGA) as the carrier, EWVDV as the targeting component, and PFH and India ink as signaling components to construct multimodal NPs (EWVDV-Ink-PFH) by a three-step emulsification method and a carbodiimide method. We examined the basic properties of the NPs and confirmed the optimal concentrations by studying the relationship between the concentration of the NPs and the US imaging and cryosection results verified the ability of the NPs to target thrombi. Based on the above research, we used low-, moderate- and high-velocity fluids to simulate the blood flow velocity in vivo and study the influence of the flow velocity on the targeting of the NPs to thrombi using multimodal imaging. This is the first study to demonstrate the relationship between the thrombus targeting of NPs and the blood flow velocity using platelet-targeting NPs for multimodal imaging. We hope these findings will serve as a reference for the design of molecular probes or targeted drug delivery systems in the context of thrombi at different sites and in different blood vessel types according to the blood flow velocity.

\section{Materials and Methods Materials}

PLGA (containing $75 \%$ lactide and $25 \%$ glycolide, with a molecular weight of 8000) was purchased from Jinan Daigang Biological Material Co., Ltd. (Shandong, China). PFH was obtained from J\&K Scientific, Ltd. (Beijing, China). India ink was purchased from Amresco (Washington, USA). EWVDV peptide modified with gallic acid was obtained from China Peptides Co., Ltd. (Shanghai, China). Polyvinyl alcohol (PVA) with an average molecular weight of 30,000-70,000, 1-ethyl-3-(3-dimethylaminopropyl) carbodiimide hydrochloride (EDC), N-hydroxysuccinimide (NHS) and 1,1'-dioctadecyl-3,3,3',3'-tetramethylindotricarbocyanine iodide (DIR) were obtained from Sigma-Aldrich Corporation (St. Louis, MO, USA). All other reagents used were of at least analytical grade.

\section{Preparation of the NPs}

A three-step emulsification process was used to prepare the Ink-PFH NPs (nontargeted NPs, NTNPs). First, 200 $\mu \mathrm{L}$ of $10 \%$ India ink was added to $300 \mu \mathrm{L}$ of $\mathrm{PFH}$. Acoustic emulsification for $70 \mathrm{~s}$ using an ultrasonic oscillation instrument (VCY-500, YYSonics Co., Ltd., Shanghai, China) with $100 \mathrm{~W}$ produced a black emulsion. 
Then, $100 \mathrm{mg}$ of PLGA was fully dissolved in $3 \mathrm{~mL}$ of dichloromethane, and the black emulsion was added to the PLGA solution and acoustically emulsified by $100 \mathrm{~W}$ for 70 s. Subsequently, $10 \mathrm{~mL}$ of $4 \%$ PVA was added and acoustically emulsified for $70 \mathrm{~s}$ to produce a light gray solution. Finally, $20 \mathrm{~mL}$ of $2 \%$ isopropanol was added and stirred continuously in an ice bath for $2 \mathrm{~h}$ until volatilization of the organic solvents occurred. The Ink-PFH NPs were washed with double-distilled water, and the supernatant was removed three times.

Carbodiimide-mediated amide bond formation was used to prepare EWVDV-Ink-PFH NPs (targeted NPs, TNPs). EWVDV is a short peptide made of Glu-Trp-Val-Asp-Val amino acids. In general, an appropriate amount of NTNPs was dispersed in 0.1 M MES buffer solution $(\mathrm{pH}=5)$. Excessive amounts of activating agents EDC and NHS with a mass ratio of 2:1 were added and incubated with the NTNPs for $1 \mathrm{~h}$. Double-distilled water was used to wash away the unreacted EDC and NHS. The activated NTNPs were dispersed in $0.1 \mathrm{M}$ MES buffer solution $(\mathrm{pH}=8)$ mixed with rhodamine-modified EWVDV peptide for 1 $\mathrm{h}$ on a shaking table and rinsed with double-distilled water to remove the unreacted EWVDV. The NPs were placed in an ice bath throughout the entire procedure, and the TNPs were collected and stored at $4{ }^{\circ} \mathrm{C}$. DiR was added to the dissolved PLGA to prepare DiR-stained NTNPs and DiRstained TNPs using the same procedures.

\section{Characterization of the NPs}

NTNPs and TNPs diluted to a transparent liquid were dropped into cuvettes and potentiometers. The size, polydispersity index (PDI), and zeta potential of the different NPs were determined at $25{ }^{\circ} \mathrm{C}$ using a laser particle size analyzer (Zetasizer Nano ZS90, Malvern Instruments, Ltd., Worcestershire, UK). Microscopic and fluorescence images of the NPs were collected by optical microscopy (Olympus CKX41, Olympus Co., Ltd., Tokyo, Japan). The loading rate of the EWVDV modified with rhodamine was measured by flow cytometry (FACSVantage SE, Becton Dickinson, San Jose, CA, USA). We randomly counted 10,000 NPs to measure the loading rate of the EWVDV.

To observe the "liquid to gas" phase transition of the NPs, $50 \mu \mathrm{L}$ of $5 \mathrm{mg} / \mathrm{mL}$ NTNPs and TNPs dispersed in double-distilled water was dropped onto glass slides for imaging by optical microscopy at room temperature $\left(23.6{ }^{\circ} \mathrm{C}\right.$, magnification $\left.4 \times 10\right)$. After heating the NPs to $70{ }^{\circ} \mathrm{C}$ using a heating plate, images were collected again at the same magnification.

\section{Multimodal Imaging of the TNPs}

To determine the appropriate concentration of TNPs for imaging, different concentrations $(2.5,5.0,7.5,10.0 \mathrm{mg} / \mathrm{mL})$ of TNPs were added to the agarose model. After $5 \mathrm{~min}$ of LIFU (Haifu Medical Technology Co., Ltd., Chongqing, China) irradiation at $4 \mathrm{~W} / \mathrm{cm}^{2}$, B mode US images and contrastenhanced ultrasound (CEUS) images were collected using an US imaging system (MyLab 90, Esaote, Italy). The acoustic intensities of the US images were recorded using grayscale quantitative analysis software (Ultrasound Molecular Imaging Institute, Chongqing, China). PA images were collected using a PA imaging system (Vevo LAZR, VisualSonics, Inc., Toronto, Canada) after irradiation for $5 \mathrm{~min}$ with a laser fluence of $2 \mathrm{~mJ} / \mathrm{cm}^{2}$ at absorption peak of $706 \mathrm{~nm}$; then, the PA intensities were measured.

\section{Thrombus-Targeting Performance of the NPs}

The animal experiments were approved by the Animal Ethics Committee of Chongqing Medical University and conducted in accordance with the guidelines of the Institutional Animal Care and Use Committee of Chongqing Medical University. To prepare the thrombus in vitro, New Zealand white rabbits $(2 \mathrm{~kg}$ ) were purchased from the Animal Center of Chongqing Medical University. Blood $(10 \mathrm{~mL})$ was collected from the ear aortas of New Zealand white rabbits and incubated in 20 -mL beakers at $37^{\circ} \mathrm{C}$ for $5 \mathrm{~h}$ for thrombus formation. The thrombus was cut into squares of $5 \mathrm{~mm} \times 5 \mathrm{~mm}$.

To observe the thrombus-targeting performance of the different NPs, $5 \mathrm{~mL}$ of $5 \mathrm{mg} / \mathrm{mL}$ NTNPs and TNPs was incubated with the thrombi for $30 \mathrm{~min}$. Saline was used to remove free NPs. Then, the thrombi were cryosectioned at $20 \mu \mathrm{m}$. The cryosections were heated to $70{ }^{\circ} \mathrm{C}$ using a heating plate, and a small amount of double-distilled water was added to the thrombus to induce the phase transition of the NPs; then, images were collected by optical microscopy at a magnification of $4 \times 10$.

Five milliliters of $5 \mathrm{mg} / \mathrm{mL}$ NTNPs and TNPs were incubated with the thrombus samples for $5 \mathrm{~min}$ in the agarose model. B mode and CEUS images were collected. Images were collected again after $180 \mathrm{~s}$ of irradiation by LIFU at a power of $4 \mathrm{~W} / \mathrm{cm}^{2}$.

\section{Extracorporeal Circulation Experiment with Thrombi}

To simulate internal circulation, precision peristaltic pumps (UIP, Kamoer, Shanghai, China), silicone tubing, 
polypropylene plastic tubing, and an ice box were used to assemble an extracorporeal circulation model (Figure 1). A thrombus $5 \mathrm{~mm} \times 5 \mathrm{~mm}$ in size was placed in the plastic tube, and a 3-mm-thick sponge was used to secure the thrombus. After $50 \mathrm{~mL}$ of $5 \mathrm{mg} / \mathrm{mL}$, NPs were inserted into the circulation model, we closed the silicone tubing of the circulation device and set a cycle time of $500 \mathrm{~s}$. TNPs, NTNPs, and saline were used to study the targeting effect of the NPs at a blood flow velocity of $0,20,40$, and $60 \mathrm{~cm} / \mathrm{s}$. The flow velocity of 0 or $20 \mathrm{~cm} / \mathrm{s}$ can occur in these situations, such as in venules, capillaries, transient flow stoppages, the anterior and posterior parts of stenotic vessels and embolized blood vessels. ${ }^{21,22}$ The flow velocity of $40 \mathrm{~cm} / \mathrm{s}$ is similar to the flow velocity in large arteries. $^{22}$ The flow velocity of $40 \mathrm{~cm} / \mathrm{s}$ is similar to that in the aorta. ${ }^{22}$ The entire process was performed in an ice bath box, and each group of experiments was repeated 3 times. After each experiment was completed, we removed the free NPs of thrombi with saline and cleaned the circulation device with double-distilled water.

To observe the targeting effect, the thrombus samples exposed to blood flow velocities of $0,20,40$, and $60 \mathrm{~cm} / \mathrm{s}$ were fixed in $20-\mathrm{mL}$ beakers with $3 \%$ agarose solution, and the agarose models were cut into cubes. PA images were collected after $5 \mathrm{~min}$ of irradiation with a 706-nm laser at a fluence of $2 \mathrm{~mJ} / \mathrm{cm}^{2}$ by PA imaging system. To collect fluorescence images of the thrombi incubated with the DiRstained NPs and saline at blood flow velocities of $0,20,40$, and $60 \mathrm{~cm} / \mathrm{s}$, the thrombi were placed in a plastic box and imaged using a small animal fluorescence imaging system (NightOwl LB983, Berthold, Germany) at an excitation wavelength of $740 \mathrm{~nm}$.

\section{Statistical Analysis}

The data were analyzed using the Statistical Program for the Social Sciences (SPSS for Windows, version 22.00, Chicago, IL, USA). Continuous variables are presented as the mean \pm the standard deviation. All experimental samples comprised no less than three replicates. Independent sample t-tests, one-way ANOVA and post-tests were used to compare differences in the fluorescence intensity of thrombi incubated with the TNPs and NTNPs. The level of significance was set to $\mathrm{P}<0.05$.

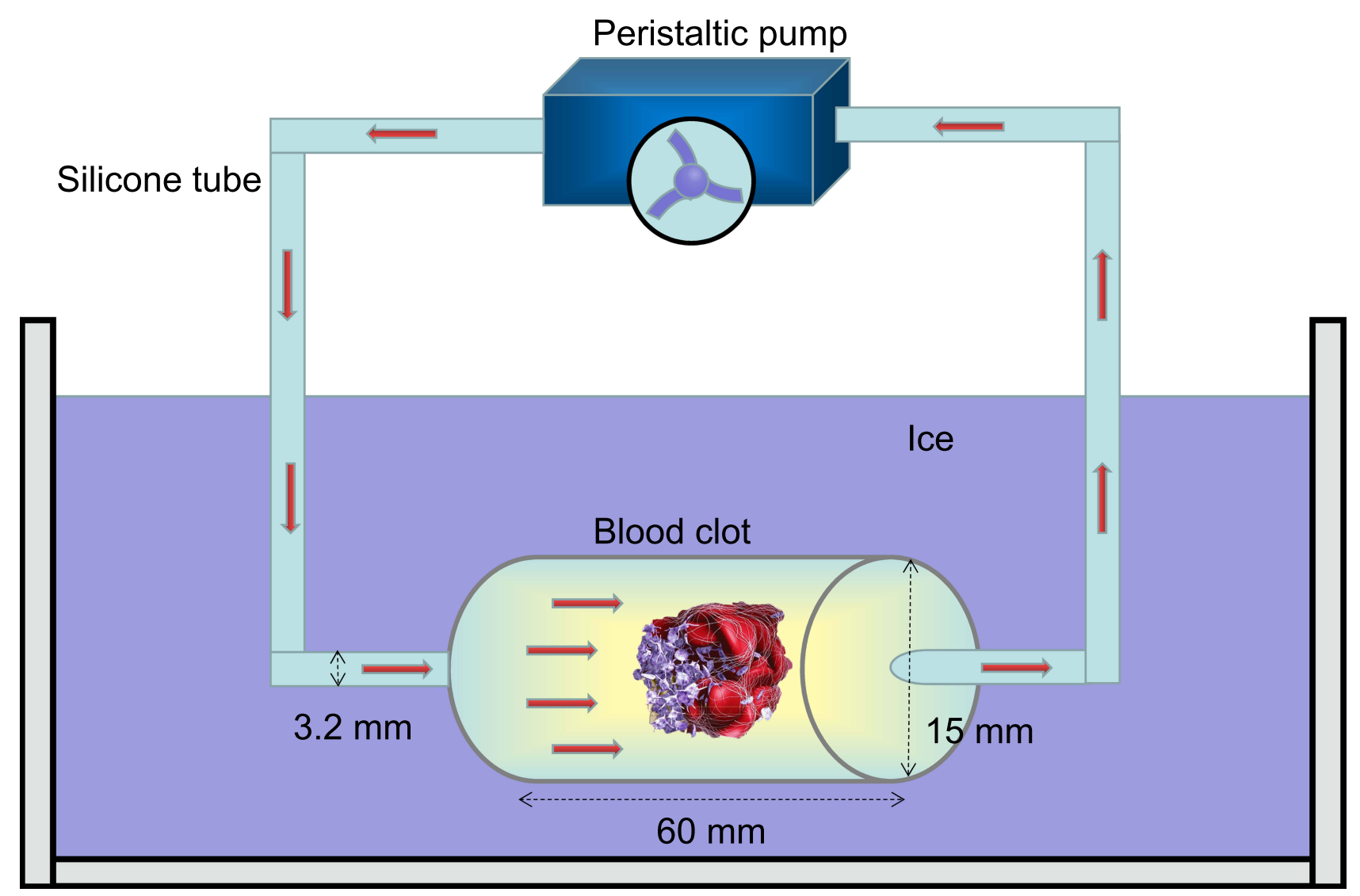

Figure I Schematic of the extracorporeal circulation device, consisting of precision peristaltic pumps, silicone tubing, polypropylene plastic tubing, and an ice box. 


\section{Results}

\section{Characterization of the NPs}

The NTNPs were $335.2 \pm 76.52 \mathrm{~nm}$ in size with a PDI of $0.179 \pm 0.061$ and a zeta potential of $-17.3 \pm 3.85 \mathrm{mV}$. The TNPs were $370.7 \pm 99.96 \mathrm{~nm}$ in size with a PDI of $0.172 \pm$
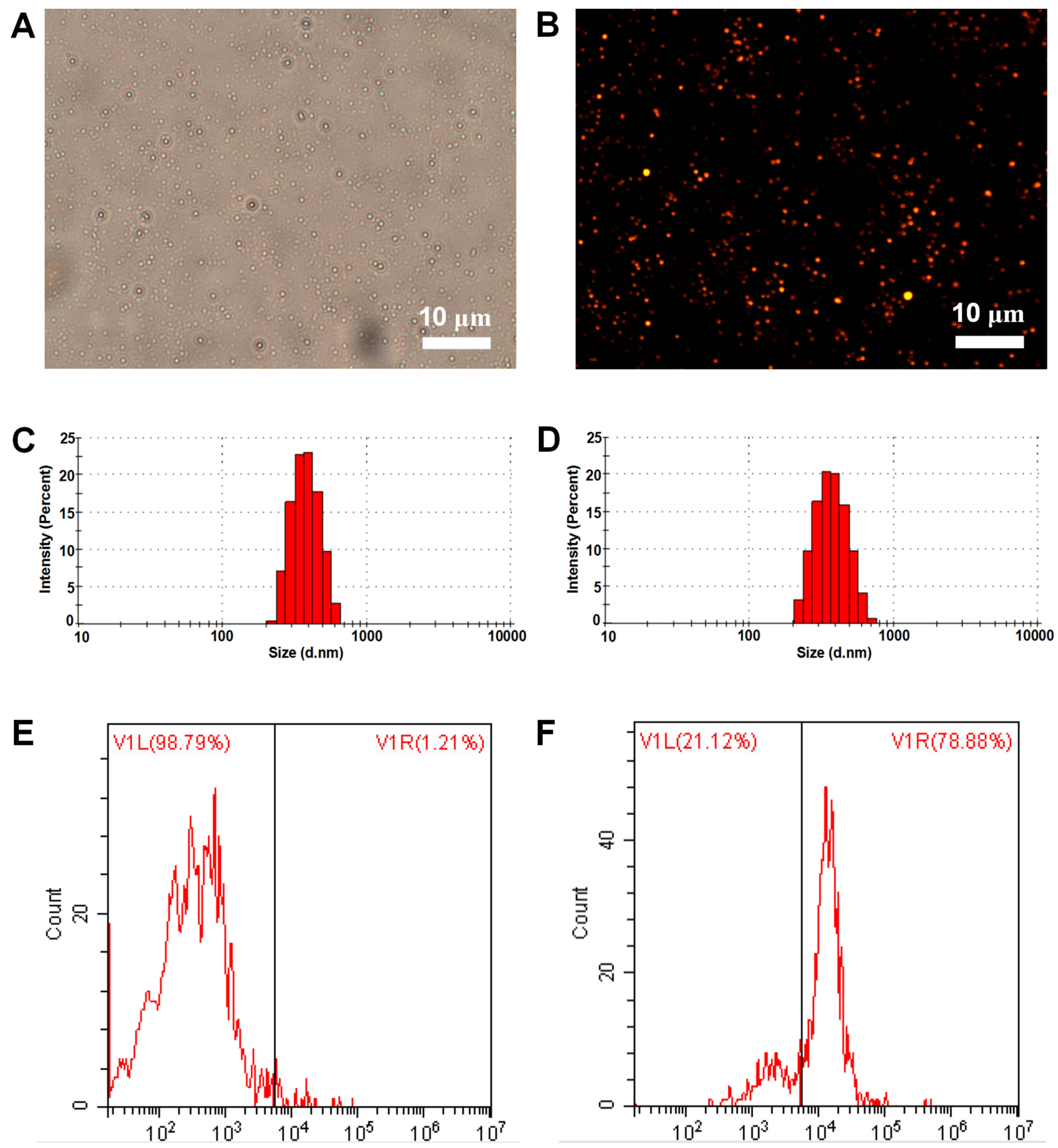

Figure 2 Characteristics of the NPs.

Notes: Optical microscopy image (A) and fluorescence microscopy image (B) of the TNPs. Size distribution by intensity of the NTNPs (C) and TNPs (D). Fluorescence distribution of the NTNPs (E) and TNPs (F).

Abbreviations: NPs, nanoparticles; TNPs, targeted nanoparticles; NTNPs, nontargeted nanoparticles. 


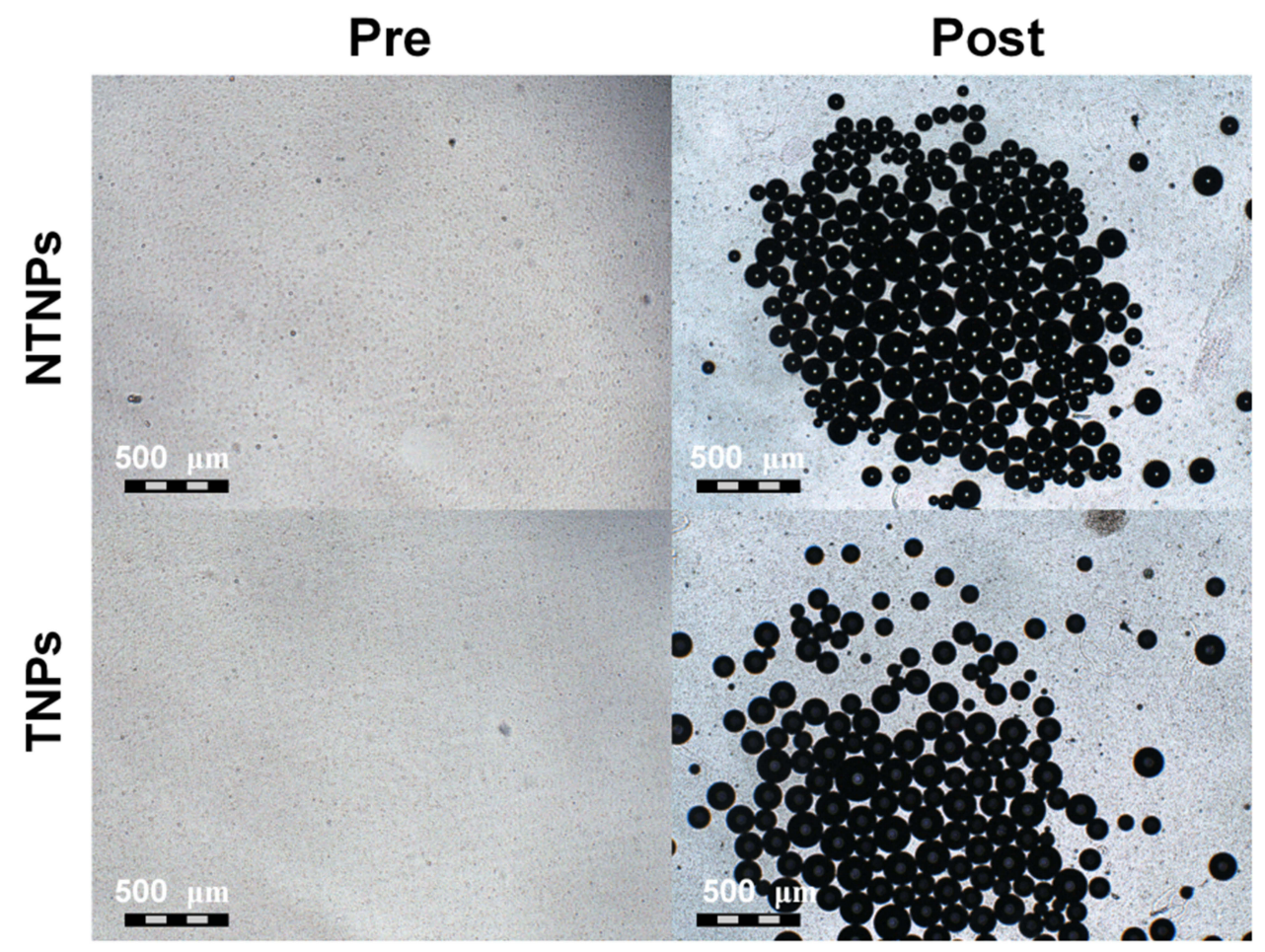

Figure 3 Thermal phase transition of the NPs.

Notes: Before heating, the NTNPs and TNPs could not be clearly displayed at a magnification of $4 \times 10$ by optical microscopy. After heating, the NTNPs and TNPs turned into microbubbles due to the phase transition, and the size of the NPs significantly increased.

Abbreviations: NPs, nanoparticles; TNPs, targeted nanoparticles; NTNPs, nontargeted nanoparticles.

EWVDV modified with rhodamine was successfully linked to the surface of the TNPs; and the loading rate of the EWVDV was $77.67 \%$.

As shown in Figure 3, before heating, the NTNPs could not be clearly displayed at a magnification of $4 \times 10$ by optical microscopy. After heating, the NTNPs turned into microbubbles via the phase transition and tended to aggregate. The volume and number of transition NPs increased during this process. The same results were observed in the TNP group.

\section{Multimodal Imaging of the TNPs}

After irradiation by LIFU, a large number of microbubbles could be observed on the surface of the solution. B mode and CEUS signals were detected in each group (Figure 4A and $\mathrm{B})$. The US signal was weak at a concentration of $2.5 \mathrm{mg} / \mathrm{mL}$, but a uniform and intense US signal were obtained at a concentration of $5.0,7.5$ and $10.0 \mathrm{mg} / \mathrm{mL}$. The acoustic intensity in the B mode and CEUS images is shown in Figure 4D and E; the intensity increased with increasing TNP concentrations.

As illustrated in Figure 4C, PA signals were detected for different concentrations of TNPs after irradiation. The
PA signal was weak at a concentration of $2.5 \mathrm{mg} / \mathrm{mL}$, but a uniform and intense PA signal was obtained at a concentration of $5.0,7.5$ and $10.0 \mathrm{mg} / \mathrm{mL}$. The PA intensity of the TNPs is shown in Figure 4F; the intensity increased with increasing TNP concentrations.

\section{Thrombus-Targeting Performance of the NPs}

In the TNP group, a large number of transitioned TNPs could be observed inside the thrombi after the addition of double-distilled water. Masses of transitioned TNPs were also found deep in the thrombi. In the NTNP group, there were small amounts of microbubbles in the thrombi (Figure 5).

As shown in Figure 6, in the TNP group, before LIFU irradiation, the thrombi were slightly hypoechoic in $\mathrm{B}$ mode and showed almost no signal on CEUS imaging, while hyperechoic signals at the edge of thrombi were observed by $\mathrm{B}$ mode and CEUS imaging after LIFU irradiation. In the NTNP group, there were weak hyperechoic signals at the edge of thrombi due to physical adsorption in B mode and on CEUS imaging after LIFU irradiation. 

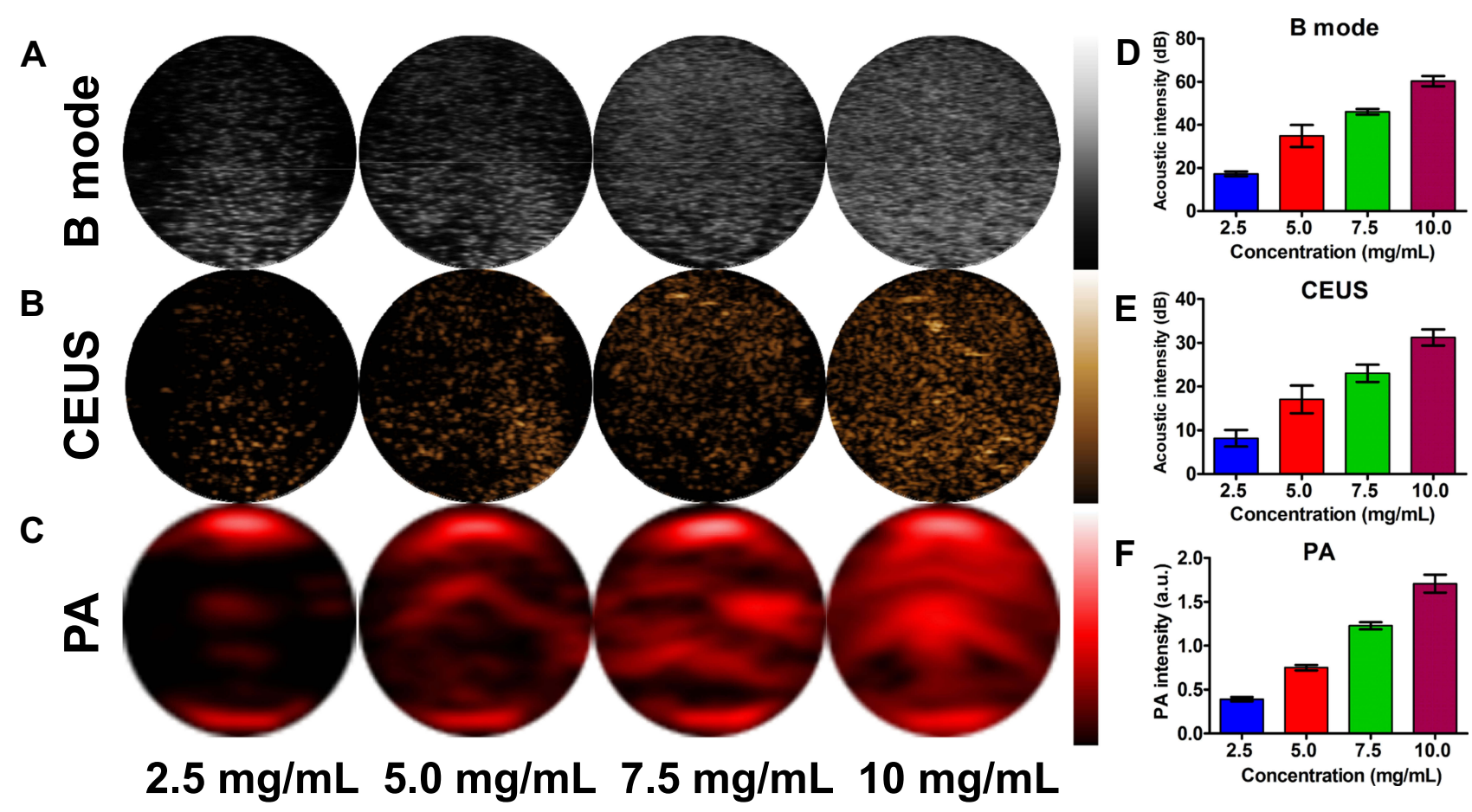

Figure 4 US and PA images of the TNPs at a concentration of $2.5,5.0,7.5$, and $10.0 \mathrm{mg} / \mathrm{mL}$.

Notes: B mode US (A) and CEUS (B) images of the TNPs after irradiation by LIFU. PA images of the TNPs (C). Acoustic intensity of the TNPs on B mode (D) and CEUS (E) imaging. PA intensity of the TNPs (F).

Abbreviations: US, ultrasound; CEUS, contrast-enhanced ultrasound; PA, photoacoustic; LIFU, low-intensity focused ultrasound; TNPs, targeted nanoparticles.

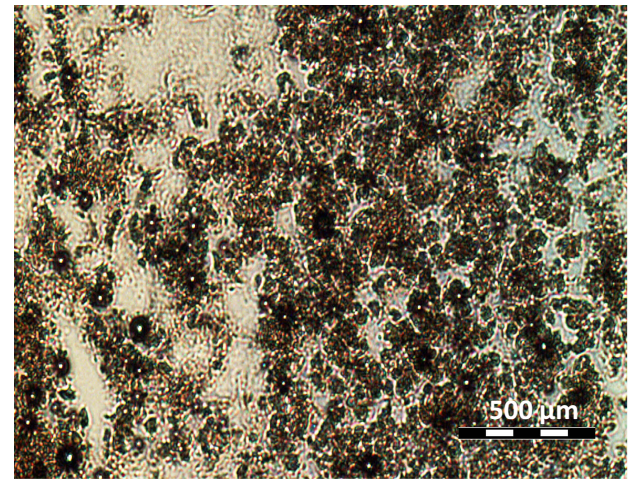

NTNPs

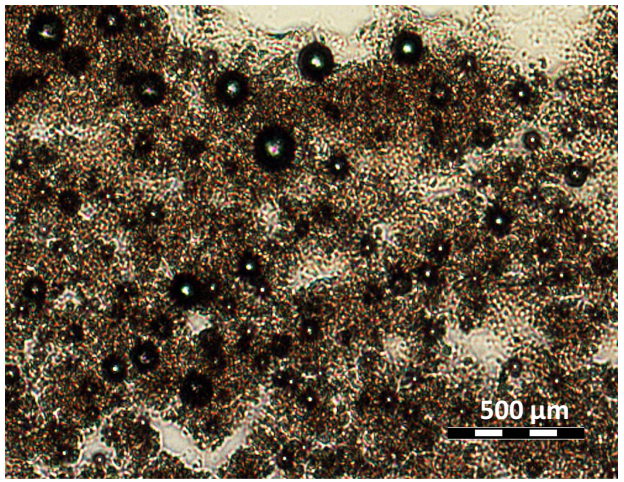

TNPs

Figure 5 Thrombus-targeting performance of the NPs.

Notes: After adding double-distilled water, a large number of transitioned TNPs could be seen inside the thrombi. However, there were small amounts of microbubbles in the thrombi in the NTNP group.

Abbreviations: NPs, nanoparticles; TNPs, targeted nanoparticles; NTNPs, nontargeted nanoparticles.

\section{Extracorporeal Circulation Experiment with Thrombi}

As shown in Figure 7, there was no red PA signal on thrombi in the saline group at different blood flow velocities. A strong red PA signal could be seen on the thrombi in the TNP group at blood flow velocities of 0,20 , and $40 \mathrm{~cm} / \mathrm{s}$, while a weak PA signal could be seen at a blood flow velocity of $60 \mathrm{~cm} / \mathrm{s}$. In the NTNP group, the thrombi showed a weak red PA signal at $0 \mathrm{~cm} / \mathrm{s}$ and no PA signal in the other blood flow velocity groups.

The fluorescence intensity of the TNPs and NTNPs at the different blood flow velocities and all statistical data are shown in Tables 1 and 2. As shown in Figure 8A, in the TNP group, strong fluorescence 


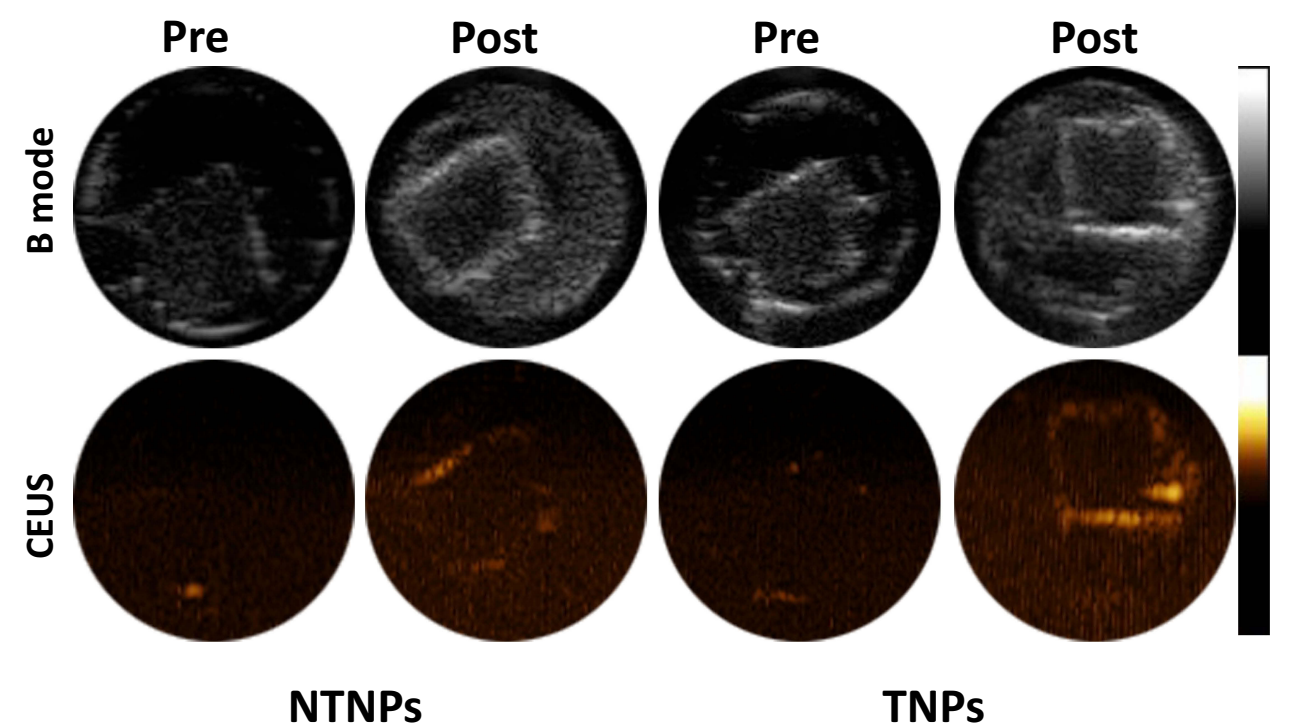

Figure 6 US images of NPs in thrombi.

Notes: In the TNP group, before LIFU irradiation, the thrombi were slightly hypoechoic in B mode and showed almost no signal on CEUS imaging, while hyperechoic signals were observed at the edge of thrombi in B mode and on CEUS imaging after LIFU irradiation at a power of 4 W/cm $\mathrm{cm}^{2}$ for $180 \mathrm{~s}$. In the NTNP group, there were weak hyperechoic signals at the edge of thrombi in B mode and on CEUS imaging due to physical adsorption after LIFU irradiation.

Abbreviations: US, ultrasound; CEUS, contrast-enhanced ultrasound; LIFU, low-intensity focused ultrasound; NPs, nanoparticles; TNPs, targeted nanoparticles; NTNPs, nontargeted nanoparticles.

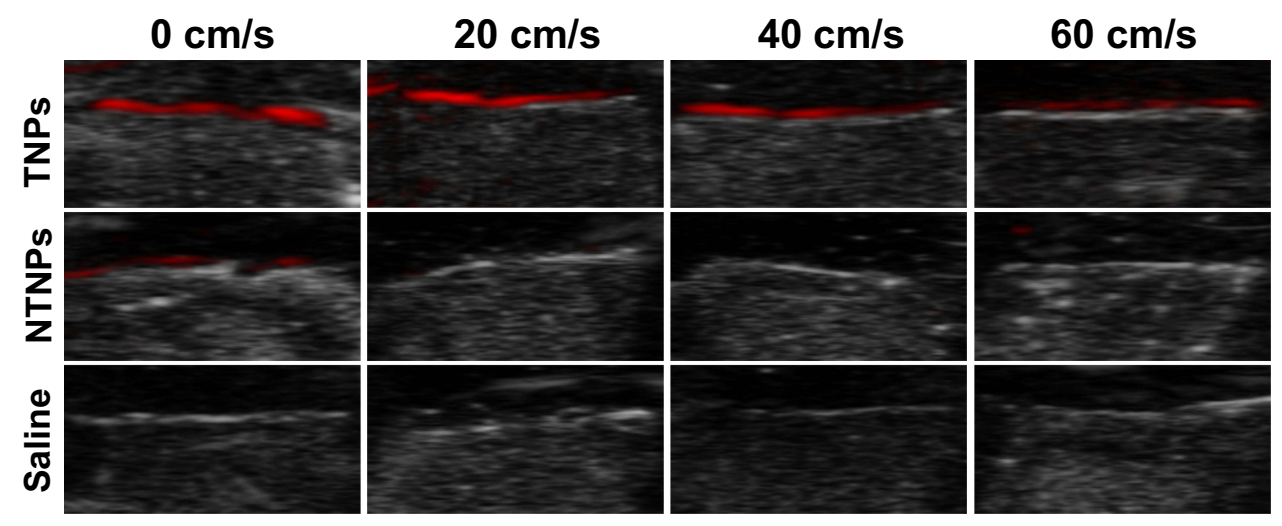

Figure 7 PA images of thrombi in the different groups at different blood flow velocities.

Notes: A strong PA signal was observed on the thrombi in the TNP group at blood flow velocities of 0,20 , and $40 \mathrm{~cm} / \mathrm{s}$, while a weak PA signal was observed at a blood flow velocity of $60 \mathrm{~cm} / \mathrm{s}$. In the NTNP group, the thrombi showed a weak PA signal at $0 \mathrm{~cm} / \mathrm{s}$ and no PA signal in the other flow velocity groups. No PA signal was observed in the saline group at any flow velocity.

Abbreviations: PA, photoacoustic; TNPs, targeted nanoparticles; NTNPs, nontargeted nanoparticles.

signals could be observed on the thrombi at blood flow velocities of 0 and $20 \mathrm{~cm} / \mathrm{s}$, and the difference in the fluorescence intensity at 0 and $20 \mathrm{~cm} / \mathrm{s}$ was not significant $(\mathrm{P}>0.05)$. The fluorescence intensity of the thrombi was reduced to a certain extent at $40 \mathrm{~cm} / \mathrm{s}$ and significantly reduced at $60 \mathrm{~cm} / \mathrm{s}$. The difference in the fluorescence intensity at $20 \mathrm{vs} 40 \mathrm{~cm} / \mathrm{s}$ and $40 \mathrm{vs}$ $60 \mathrm{~cm} / \mathrm{s}$ was significant (both $\mathrm{P}<0.05$ ). In the NTNP group, the thrombi showed weak fluorescence at $0 \mathrm{~cm} / \mathrm{s}$, and the fluorescence intensity of the thrombus gradually decreased with increasing blood flow velocity (Figure $8 \mathrm{~B}$ ). There was no fluorescence signal on the thrombi in the saline group at any blood flow velocity (Figure 8C). At the same blood flow velocity, the fluorescence intensity in the TNP group was significantly higher than that in the NTNP group (all $\mathrm{P}<0.05$ ). Fluorescence surface images of the TNP and NTNP thrombi are shown in Figure 8D and E. The fluorescence intensity of the TNP and NTNP thrombi at different blood flow velocities is shown in Figure 8F. 
Table I Fluorescence Intensity of Thrombi in the TNP and NTNP Groups at Different Flow Velocities

\begin{tabular}{|l|l|l|l|}
\hline $\begin{array}{l}\text { Flow Velocity } \\
(\mathbf{c m} / \mathbf{s})\end{array}$ & TNPs (cps) & NTNPs (cps) & p-values \\
\hline 0 & $113,611 \pm 8105$ & $22,032 \pm 1608$ & $<0.000 \mathrm{I}$ \\
20 & $115,50 I \pm 7053$ & $18,448 \pm 303$ & $<0.000 \mathrm{I}$ \\
40 & $53,82 I \pm 6698$ & $15,060 \pm 122$ & $<0.000 \mathrm{I}$ \\
60 & $18,792 \pm 100$ & $940 I \pm 204$ & $<0.000 \mathrm{I}$ \\
P-values & 0.0175 & $<0.000 \mathrm{I}$ & \\
\hline
\end{tabular}

Abbreviations: TNPs, targeted nanoparticles; NTNPs, nontargeted nanoparticles; cps, cards per second.

Table 2 Comparison of the Fluorescence Intensity of Thrombi in the TNP and NTNP Groups at Different Flow Velocities

\begin{tabular}{|l|l|l|}
\hline $\begin{array}{l}\text { Flow Velocity } \\
(\mathbf{c m} / \mathbf{s})\end{array}$ & p-values of TNPs & p-values of NTNPs \\
\hline 0 vs 20 & 0.724 & 0.003 \\
0 vs 40 & $<0.0001$ & $<0.0001$ \\
0 vs 60 & $<0.0001$ & $<0.0001$ \\
20 vs 40 & $<0.0001$ & 0.004 \\
20 vs 60 & $<0.0001$ & $<0.0001$ \\
40 vs 60 & $<0.0001$ & $<0.0001$ \\
\hline
\end{tabular}

Abbreviations: TNPs, targeted nanoparticles; NTNPs, nontargeted nanoparticles.

\section{Discussion}

The blood flow velocity refers to the linear velocity at which a particle in the blood moves within the vessel. When blood flows in a vessel, the flow velocity is proportional to the blood flow and inversely proportional to the cross-sectional area of the blood vessel. Therefore, the blood flow velocity varies greatly in different parts of the human body. When NPs enter blood vessels, they simulate leukocytes in being captured by vascular endothelial cells, rolling, decelerating, and adhering to the surface of a thrombus. ${ }^{23}$ This process is closely related to blood flow velocity. In this study, we demonstrate for the first time the relationship between the targeting effect of NPs to thrombi and the blood flow velocity using NPs for multimodal imaging.

After coupling with EWVDV, the size of the TNPs increased from $335.2 \pm 76.52$ to $370.7 \pm 99.96 \mathrm{~nm}$, the zeta potential changed from $-17.3 \pm 3.85$ to $-24.7 \pm 4.25$ $\mathrm{mV}$, and greater red fluorescence was observed on the surface of the TNPs. These results indicate that the EWVDV peptide was successfully linked to the surface of the NTNPs. The PDI of the NTNPs and TNPs was $0.179 \pm 0.061$ and $0.172 \pm 0.082$, respectively, demonstrating that the two types of NPs have a narrow size distribution. The small and uniform particle size of NPs makes it difficult to be captured by the reticuloendothelial system, increasing their blood circulation duration. ${ }^{17}$ Through thermal phase transition experiments, we found that the TNPs and NTNPs could undergo a liquid-gas phase transition after heating, which is the basis of US imaging. Next, we studied the effects of TNPs at different concentrations by US and PA imaging and selected $5 \mathrm{mg} / \mathrm{mL}$ as the optimal concentration. The above results indicate that we successfully constructed TNPs with the ability to facilitate multimodal imaging.

Undoubtedly, the effective binding of NPs to thrombi is key to the molecular imaging of thrombotic disease. ${ }^{2}$ Platelets play a key role in the process of thrombosis and are the main component of thrombi. P-selectin is a critical component of activated platelets; upon platelet activation, it translocates to the cell surface within seconds and is highly expressed for a long time on activated platelets. ${ }^{24,25}$ These features make P-selectin an ideal target for thrombotic molecular probes. Regarding ligands, peptides are an attractive targeting component due to their relatively small size, ease of production, low cost, low immunogenicity and long-term stability. ${ }^{26}$ Appeldoorn et al reported that the EWVDV peptide has a high affinity and specificity for P-selectin, and the targeting ability is improved by 800 times after gallic acid modification. ${ }^{27}$ Therefore, EWVDV was selected as the targeting component in our research. Our targeting experiments show that a large number of transitioned TNPs could be seen inside the thrombi, as well as some microbubbles deep within the thrombus, indicating that the TNPs have good targeting and penetration capabilities. Good targeting and penetration allow greater TNP accumulation per unit volume of thrombus, which is beneficial for imaging and thrombolysis. Strong US signals were visible on the thrombi, demonstrating that the EWVDVmediated TNPs combined with LIFU-induced phase transition technology have the ability to target thrombi for US imaging.

In the circulation experiment, in the NTNP group, as the flow velocity increased, the fluorescence intensity of the thrombus gradually decreased, indicating that blood flow affects the binding of NTNPs due to physical adsorption. Under the guidance of EWVDV, the fluorescence intensities of all thrombi in the TNP group at the same flow velocity were significantly higher than those of thrombi in the NTNP group (all $\mathrm{P}<0.05$ ), demonstrating that a significantly increased concentration of TNPs at thrombi compared with NTNPs. In the TNP group, at 


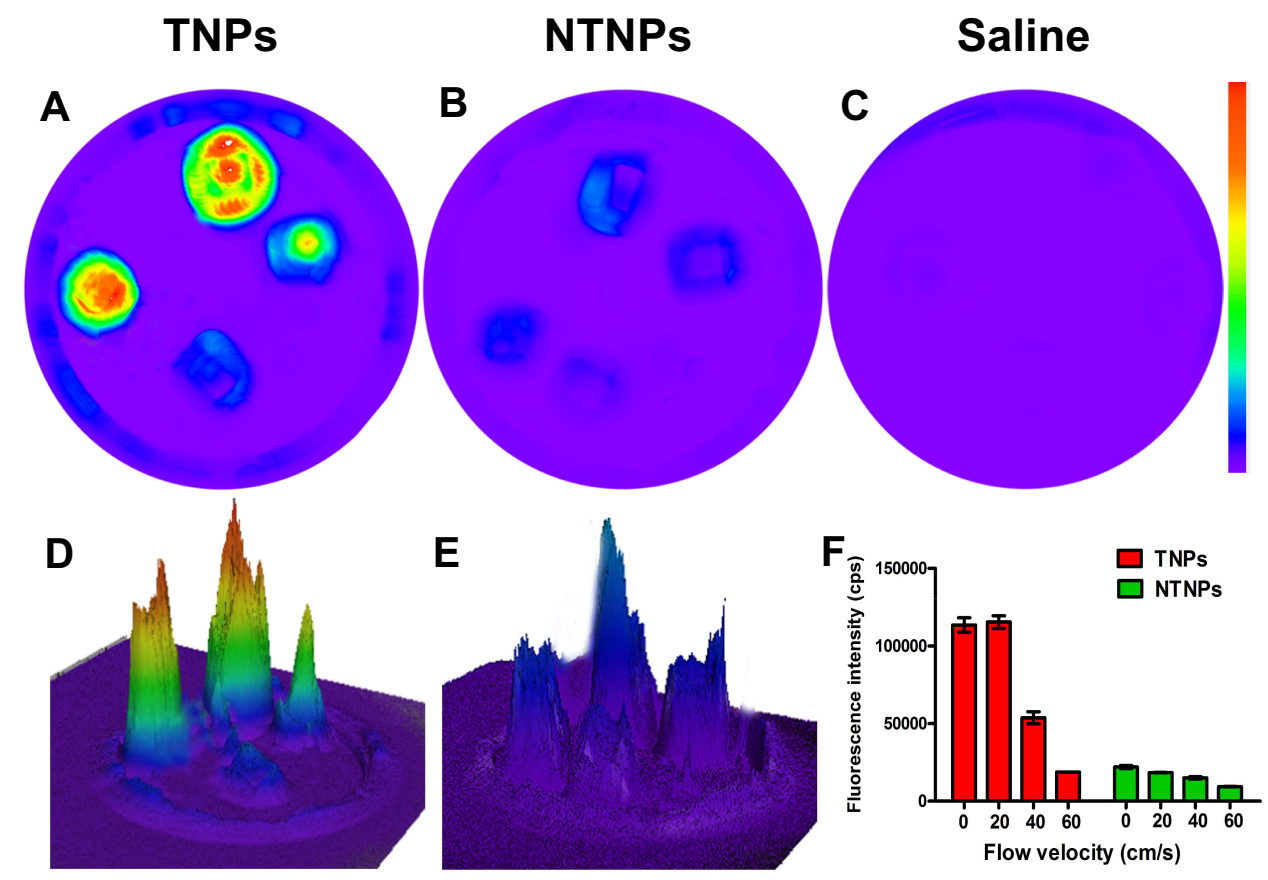

Figure 8 Fluorescence images of thrombus in the different groups at different blood flow velocities.

Notes: The blood flow velocity was $0 \mathrm{~cm} / \mathrm{s}, 20 \mathrm{~cm} / \mathrm{s}, 40 \mathrm{~cm} / \mathrm{s}$, and $60 \mathrm{~cm} / \mathrm{s}$ for the upper, left, right and lower thrombus, respectively. In the TNP group, strong fluorescence intensity on the thrombi were observed at flow velocities of 0 and $20 \mathrm{~cm} / \mathrm{s}$, and the fluorescence intensity of the thrombi at $40 \mathrm{~cm} / \mathrm{s}$ was reduced by $52.6 \%$ comparing to static fluid but were significantly reduced by $83.5 \%$ at $60 \mathrm{~cm} / \mathrm{s}(\mathbf{A})$. In the NTNP group, the thrombi showed weak fluorescence at $0 \mathrm{~cm} / \mathrm{s}$, and the fluorescence intensity gradually decreased with increasing flow velocity (B). In the saline group, there were no fluorescence signals on the thrombi at any flow velocity (C). Fluorescence surface images of thrombi in the TNP (D) and NTNP (E) groups. Fluorescence intensity of thrombi in the TNP and NTNP groups for different flow velocities (F).

Abbreviations: TNPs, targeted nanoparticles; NTNPs, nontargeted nanoparticles.

a low flow velocity $(20 \mathrm{~cm} / \mathrm{s})$, strong fluorescence was detected on the thrombus, and the fluorescence intensity was not significantly different from that in the $0 \mathrm{~cm} / \mathrm{s}$ group. This demonstrates that low flow velocities hardly affect the targeting effect of the TNPs. One reason may be that the low flow velocity exerts less shear force on the TNPs, and the TNPs roll slowly on the surface of the thrombus. Therefore, the targeting component of the TNPs has sufficient time to bind to the thrombus, thus achieving effective targeted delivery. Low or static blood flow can occur in these situations, such as in venules, capillaries, transient flow stoppages, the anterior and posterior parts of stenotic vessels and embolized blood vessels. $^{21,22}$ When designing thrombus probes or targeted drug delivery systems for these sites or situations, the blood flow velocity is not a major factor to consider. The moderate flow velocity used $(40 \mathrm{~cm} / \mathrm{s})$ is similar to the flow velocity in large arteries. ${ }^{22}$ The fluorescence intensity of the thrombi in this group was reduced by $52.6 \%$ comparing to static fluid $(0 \mathrm{~cm} / \mathrm{s})$, indicating that some of the TNPs were affected by this blood flow velocity. The high flow velocity used $(60 \mathrm{~cm} / \mathrm{s})$ is similar to that in the aorta. $^{22}$ The fluorescence intensity of thrombi in this group was significantly reduced by $83.5 \%$. The same result was observed on PA imaging. We speculate that the poor targeting effect of the TNPs may be due to the high flow velocity, which led to the rapid rolling of TNPs on the surface of the thrombi and a short contact time between the EWVDV peptide with the P-selectin on the surface of activated platelets. Before the TNPs could firmly combine with the thrombus, they were washed away. In addition, the forces associated with the interaction between the EWVDV-modified TNPs and activated platelets are not sufficient to overwhelm the shear forces generated by high-velocity blood flow. Thus, ligands that bind rapidly and stably to targets are particularly important for molecular probes for thrombi in the aorta and vascular stenosis.

This study has several limitations. First, due to the limitations of the circulation model, some NPs underwent a phase transition during the experiment, which may affect the targeting effect of the NPs on US imaging. Therefore, we have only demonstrated the ability of the NPs to enable the US imaging of thrombi and did not study the relationship between the targeting effect and flow velocity by US imaging. Second, the thrombus was in a static fluid before the circulation model was run and after the model was shut 
down, and although we tried to minimize this period of the time, it unavoidably had an impact on the experimental results. Third, we only examined a NP concentration of $5 \mathrm{mg} / \mathrm{mL}$, and the targeting effect of different concentrations of NPs should be studied further. Fourth, we used rabbit blood to construct a thrombus model in this study. There are certain differences in cell composition comparing with human blood. In the future study, we will use human blood thrombus model to further study its targeting effect for the different thrombus types at different blood flow velocities. Fifth, we did not consider changes in the blood flow velocity in areas of vascular stenosis. In normal blood vessels, flow throughout most of the vasculature is laminar. When plaque formation and thrombosis cause stenosis of the vessel, the laminar flow condition is destroyed, and turbulence and vortices occur. ${ }^{21}$ The blood flow velocity varies greatly in different areas of vascular stenosis, and the complex hemodynamic environment of stenotic areas will significantly affect the adhesion of NPs. The effect of the blood flow velocity in stenotic areas on the targeting effect of NPs will be studied in future research.

\section{Conclusion}

In this study, we successfully constructed platelet-targeting NPs (EWVDV-Ink-PFH) capable of a phase transition for the multimodal imaging of thrombi, which can thus provide rich information on thrombi in real time. These targeted NPs can resist the shear forces of low blood flow velocities but are significantly affected by high blood flow velocities, particularly those occurring in the aorta and vascular stenosis. Overall, this study demonstrates the relationship between the targeting effect of NPs to thrombi and the flow velocity by multimodal imaging, which can serve as a reference for the design of NPs for thrombi at different sites and in different blood vessel types according to the blood flow velocity.

\section{Acknowledgments}

The authors are grateful to American Journal Experts (AJE) for their assistance with language editing. This research was funded by the National Natural Science Foundation of China (grant nos. 81571663, 81901807 and 81971608).

\section{Disclosure}

The authors report no conflicts of interest in this work.

\section{References}

1. Wang H, Naghavi M, Allen C, et al. Global, regional, and national life expectancy, all-cause mortality, and cause-specific mortality for 249 causes of death, 1980-2015: a systematic analysis for the global burden of disease study 2015. Lancet. 2016;388:1459-1544. doi:10.1016/S0140-6736(16)31012-1

2. Wang X, Peter K. Molecular imaging of atherothrombotic diseases: seeing is believing. Arterioscler Thromb Vasc Biol. 2017;37:10 29-1040. doi:10.1161/ATVBAHA.116.306483

3. Liu J, $\mathrm{Xu} \mathrm{J}$, Zhou J, et al. $\mathrm{Fe}_{3} \mathrm{O}_{4}$-based PLGA nanoparticles as MR contrast agents for the detection of thrombosis. Int J Nanomedicine. 2017;12:1113-1126. doi:10.2147/IJN.S123228

4. Oliveira BL, Blasi F, Rietz TA, et al. Multimodal molecular imaging reveals high target uptake and specificity of ${ }^{111} \mathrm{In}$ - and ${ }^{68} \mathrm{Ga}$-labeled fibrin-binding probes for thrombus detection in rats. $J$ Nucl Med. 2015;56:1587-1592. doi:10.2967/jnumed.115.160754

5. Wang X, Yannik G, Jathushan P, et al. Thrombus-targeted theranostic microbubbles: a new technology towards concurrent rapid ultrasound diagnosis and bleeding-free fibrinolytic treatment of thrombosis. Theranostics. 2016;6:726-738. doi:10.7150/thno.14514

6. Kim JY, Ju HR, Schellingerhout D, et al. Direct imaging of cerebral thromboemboli using computed tomography and fibrin-targeted gold nanoparticles. Theranostics. 2015;5:1098-1114. doi:10.7150/thno.11679

7. Steinmerlob AF, Kessinger CW, Erdem SS, et al. Blood accessibility to fibrin in venous thrombosis is thrombus age-dependent and predicts fibrinolytic efficacy: an in vivo fibrin molecular imaging study. Theranostics. 2015;5:1317-1327. doi:10.7150/thno.12494

8. Cui C, Yang Z, Hu X, et al. Organic semiconducting nanoparticles as efficient photoacoustic agents for lightening early thrombus and monitoring thrombolysis in living mice. ACS Nano. 2017;11:32 98-3310. doi:10.1021/acsnano.7b00594

9. Song Y, Huang Z, Xu J, et al. Multimodal SPION-CREKA peptide based agents for molecular imaging of microthrombus in a rat myocardial ischemia-reperfusion model. Biomaterials. 2014;35:2961-29 70. doi:10.1016/j.biomaterials.2013.12.038

10. Kornmann LM, Reesink KD, Reneman RS, et al. Critical appraisal of targeted ultrasound contrast agents for molecular imaging in large arteries. Ultrasound Med Biol. 2010;36:181-191. doi:10.1016/j. ultrasmedbio.2009.09.009

11. Myerson JW, Anselmo AC, Liu Y, et al. Non-affinity factors modulating vascular targeting of nano- and microcarriers. Adv Drug Deliv Rev. 2016;99:97-112. doi:10.1016/j.addr.2015.10.011

12. Feghhi S, Sniadecki NJ. Mechanobiology of platelets: techniques to study the role of fluid flow and platelet retraction forces at the micro-and nano-scale. Int J Mol Sci. 2011;12:9009-9030. doi:10.33 90/ijms12129009

13. Modery CL, Ravikumar M, Wong TL, et al. Heteromultivalent liposomal nanoconstructs for enhanced targeting and shear-stable binding to active platelets for site-selective vascular drug delivery. Biomaterials. 2011;32:9504-9514. doi:10.1016/j.biomaterials.2011.08.067

14. Shi X, Yang J, Huang J, et al. Effects of different shear rates on the attachment and detachment of platelet thrombi. Mol Med Rep. 2016;13:2447-2456. doi:10.3892/mmr.2016.4825

15. Kyoden T, Akiguchi S, Tajiri T, et al. Three-dimensional imaging of absolute blood flow velocity and blood vessel position under low blood flow velocity based on Doppler signal information included in scattered light from red blood cells. J Appl Phys. 2017;122:194701194708. doi: $10.1063 / 1.4994080$

16. Rose MJ, Jarvis K, Chowdhary V, et al. Efficient method for volumetric assessment of peak blood flow velocity using 4D flow MRI. J Magn Reson Imaging. 2016;44:1673-1682.

17. Zhou Y, Wang Z, Chen Y, et al. Microbubbles from gas-generating perfluorohexane nanoemulsions for targeted temperature-sensitive ultrasonography and synergistic HIFU ablation of tumors. $A d v$ Mater. 2013;25:4123-4130. doi:10.1002/adma.201301655 
18. Jian J, Liu C, Gong Y, et al. India ink incorporated multifunctional phase-transition nanodroplets for photoacoustic/ultrasound dual-modality imaging and photoacoustic effect based tumor therapy. Theranostics. 2014;4:1026-1038. doi:10.7150/thno.9754

19. Xu J, Zhou J, Zhong Y, et al. Phase transition nanoparticles as multimodality contrast agents for the detection of thrombi and targeting thrombolysis: in vitro and in vivo experiments. ACS Appl Mater Interfaces. 2017;9:42525-42535. doi:10.1021/acsami.7b12689

20. Shinde Patil VR, Campbell CJ, Yun YH, et al. Particle diameter influences adhesion under flow. Biophys J. 2001;80:1733-1743.

21. Hathcock JJ. Flow effects on coagulation and thrombosis. Arterioscler Thromb Vasc Biol. 2006;26:1729-1737.

22. Marieb Elaine N, Hoehn K. The Cardiovascular System: Blood Vessels Human Anatomy \& Physiology. 9th ed. New Jersey, USA: Pearson Education; 2013:712.
23. Lindner JR. Contrast ultrasound molecular imaging of inflammation in cardiovascular disease. Cardiovasc Res. 2009;84:182-189. doi:10. 1093/cvr/cvp302

24. Merten M, Thiagarajan P. P-selectin expression on platelets determines size and stability of platelet aggregates. Circulation. 2000; 102:1931-1936.

25. Merten M, Thiagarajan P. P-selectin in arterial thrombosis. Z Kardiol. 2004;93:855-863. doi:10.1007/s00392-004-0146-5

26. Wen AM, Wang Y, Jiang K, et al. Shaping bio-inspired nanotechnologies to target thrombosis for dual optical-magnetic resonance imaging. J Mater Chem B. 2015;3:6037-6045. doi:10.1039/C5TB00879D

27. Appeldoorn CC, Molenaar TJ, Bonnefoy A, et al. Rational optimization of a short human P-selectin-binding peptide leads to nanomolar affinity antagonists. $J$ Biol Chem. 2003;278:10201-10207. doi:10.10 74/jbc.M209267200

\section{Publish your work in this journal}

The International Journal of Nanomedicine is an international, peerreviewed journal focusing on the application of nanotechnology in diagnostics, therapeutics, and drug delivery systems throughout the biomedical field. This journal is indexed on PubMed Central, MedLine, CAS, SciSearch ${ }^{\circledR}$, Current Contents ${ }^{\circledR} /$ Clinical Medicine,
Journal Citation Reports/Science Edition, EMBase, Scopus and the Elsevier Bibliographic databases. The manuscript management system is completely online and includes a very quick and fair peer-review system, which is all easy to use. Visit http://www.dovepress.com/ testimonials.php to read real quotes from published authors. 